\title{
CREACIÓN DE UN MODELO DE ANÁLISIS \\ PARA LA IDENTIFICACIÓN DE LA PRESENCIA/AUSENCIA DE ATENUACIÓN EN UN CORPUS DE ADQUISICIÓN DEL ESPAÑOL DE ESTUDIANTES FRANCESES
}

\author{
CREATION OF A MODEL OF ANALYSIS TO IDENTIFY \\ THE PRESENCE/ABSENCE OF MITIGATION IN A CORPUS OF DISCOURSE \\ PRODUCED BY FRENCH LEARNERS OF SPANISH
}

\author{
DANiEl SECChi \\ Universitat de València / Université de Picardie Jules Verne \\ daniel.secchi@yahoo.es
}

Recibido: 01/05/2017

Aceptado: 21/06/2017

\begin{abstract}
Resumen
Este artículo pretende destacar la importancia de la enseñanza de la atenuación como estrategia pragmática que constituye una herramienta esencial para todo hablante que quiera aprender a desenvolverse en contextos comunicativos reales al igual que los nativos.

A tal fin, en este estudio analizamos un corpus real propio, a través de una ficha de análisis, con el objetivo de reconocer y clasificar cómo los estudiantes franceses usan estos mecanismos atenuantes y también la carencia, ausencia o uso inadecuado de las tácticas atenuantes, en los intercambios comunicativos analizados.

PALABRAS CLAVE: atenuación, español lengua extranjera (E/LE), modelo de análisis, debates y conversaciones argumentativas.
\end{abstract}

\begin{abstract}
The present paper aims to highlight the importance of teaching mitigation as a pragmatic strategy, which represents an essential tool for every learner who wants to speak as well as a native speaker in real communicative contexts. For that purpose, in the present investigation, we will analyse our own real corpus thanks to the use of a table of analysis to identify and classify how French students manage the use of mitigation strategies. At the same time, we will study the lack, the absence or the incorrect use of mitigation tactics in all the communicative exchanges that will be analysed.

KEYWORDS: mitigation, Spanish as foreign language (SFL), analysis model, debates and argumentative conversations.
\end{abstract}

Para citar este artículo / To cite this article: Secchi, Daniel (2018): Creación de un modelo de análisis para la identificación de la presencia/ausencia de atenuación en un corpus de adquisición del español de estudiantes franceses. García Ramón, Amparo y Soler Bonafont, María Amparo (Eds.): ELUA: Estudios de atenuación en el discurso, Anexo IV, págs. 303-322.

Enlace / Link: http://dx.doi.org/10.14198/ELUA2018.Anexo4.17 


\section{INTRODUCCIÓN}

En las últimas décadas, las investigaciones sobre la atenuación han ido cobrando cada vez más importancia, dando lugar a la publicación de numerosos trabajos con diferentes enfoques, entre ellos los de Lakoff (1973), Fraser (1980) y Bazzanella, Caffi y Sbisà (1991). En la actualidad, uno de los estudios más recientes orientados a la investigación de la atenuación es el proyecto Es.Var.Atenuación ${ }^{1}$. Este proyecto ha sido diseñado con el objetivo de realizar un análisis contrastivo de la atenuación pragmática en el español hablado, teniendo en cuenta las variaciones diafásicas y diatópicas del español de España y de América. Para el análisis de estos mecanismos atenuadores, se ha diseñado una ficha metodológica (Albelda, Briz, Cestero, Kotwica y Villalba 2014b), que también facilita el reconocimiento y la clasificación de los elementos extraídos. A lo largo de los años, diferentes autores han empleado otros términos con referencia al concepto de atenuación, aunque, a veces, con otras delimitaciones: Lakoff habla de deintensification y de heging, Fraser y Leech hablan de mitigation y Held habla de minimization.

En nuestro trabajo, para referirnos a esta categoría pragmática, hemos elegido el término atenuación utilizado por Albelda y Briz. Para estos autores, la atenuación no solamente regula las relaciones sociales e interpersonales entre los participantes a la interacción comunicativa, sino que constituye "una categoría pragmática cuya función consiste en minimizar la fuerza ilocutiva de los actos de habla" (Albelda y Briz, 2010: 238). Por esta razón los participantes de la enunciación utilizan la atenuación para proteger su imagen, para prevenir un posible daño a la propia imagen y salvaguardar la imagen de sus interlocutores o para reparar un daño a la propia imagen o a la imagen de ambos (Albelda et alii 2014). Además, los autores afirman que la atenuación es un "mecanismo táctico, que tiene que ver con la efectividad y la eficacia del discurso, con el logro de los fines en la interacción, además de tratarse de una función solo determinable contextualmente" (Briz y Albelda 2013: 292). Esta definición enlaza con las definiciones de otros autores como Fraser (1980 y 1990), Caffi (1999), Meyer-Hermann (1988) y Briz (2007).

El presente trabajo es la base de partida para el análisis de un corpus propio (inédito), que forma parte de un trabajo más extenso (nuestra tesis doctoral), y que tiene como objetivo el análisis para la identificación de la presencia/ausencia y la clasificación de los mecanismos de atenuación empleados por un grupo de estudiantes universitarios franceses aprendices de Español como Lengua Extranjera (en adelante E/LE).

En este trabajo presentamos los pasos que hemos seguido para llegar a la creación de una ficha de análisis que nos permite examinar un corpus proprio constituido por veintitrés grabaciones y transcripciones de debates y conversaciones argumentativas de estudiantes universitarios franceses de E/LE. En este artículo nos limitaremos a detallar los pasos que hemos dado para la creación, modificación y adaptación de la ficha de análisis para el estudio de en una primera sección del corpus constituida por nueve de las veintitrés grabaciones y transcripciones de debates y conversaciones argumentativas de la cuales: tres formales, tres semiformales y tres informales.

A continuación, tras una breve presentación del estado de la cuestión y tras haber justificado la importancia de incluir el estudio de la atenuación en E/LE, se detallan los parámetros

1 Investigadora Principal: Dr. ${ }^{a}$ Marta Albelda Marco (Universitat de València). 
que han sido elegidos y extraídos a partir de la ficha ES.VAR.ATENUACIÓN y se describen los pasos que hemos dado para llegar a la creación de tres nuevas aportaciones. Estas nuevas aportaciones han sido implementadas a la ficha de análisis ES.VAR.ATENUACIÓN como solución específica para la identificación y clasificación de la presencia/ausencia de elementos atenuantes en las interacciones del grupo de estudiantes franceses que hemos analizados.

\section{BREVE ESTADO DE LA CUESTIÓN}

Como señalábamos anteriormente, el punto de partida es una aproximación pragmática a la atenuación como categoría estratégica dirigida a la negociación comunicativa (Briz 2006, Briz y Albelda 2013). La expresión de este fenómeno pragmático puede llevarse a cabo a partir de diversos mecanismos fónicos, morfosintácticos, léxico-semánticos y discursivos, cuyo valor pragmático solo podrá ser reconocido en un contexto de uso. En este sentido, tras revisar la nómina de los mecanismos atenuantes en el Plan Curricular del Instituto Cervantes (Instituto Cervantes 2006), hemos procedido con el análisis de nuestro corpus propio (inédito) de estudiantes universitarios franceses aprendices de E/LE, de nivel B1-C1, para poder analizar las formas, las funciones y los elementos atenuantes utilizados por los aprendices en los intercambios comunicativos en los que han sido grabados.

\section{3. ¿POR QUÉ ATENUACIÓN EN LA ENSEÑANZA DEL ESPAÑOL COMO LEN- GUA EXTRANJERA?}

De acuerdo con el autor Sánchez Lobato (1999: 9), somos conscientes de que “dos o más individuos de la misma o diferente cultura pueden dominar el mismo código lingüístico, pero si no poseen parecidos conocimientos socioculturales (tanto el hablante como el oyente), difícilmente podrán compartir los mismos presupuestos y los mismos sobreentendidos".

Es por ello que una de las primeras finalidades de la introducción de la atenuación en E/ LE a través de corpus reales es la de sensibilizar a los estudiantes con el concepto de atenuación como estrategia comunicativa. Gracias a ello, se pretende que los discentes aprendan a utilizar las formas lingüísticas adecuadas a cada función comunicativa tal y como lo haría un nativo español, sin dejar al azar el logro de sus objetivos comunicativos.

Puesto que también en su lengua materna (el francés) utilizan estas estrategias de forma inconsciente para lograr sus objetivos, llevar la atenuación al aula E/LE permitiría un doble estudio, esto es, permitiría que los estudiantes pasen de un aprendizaje inductivo del español a un aprendizaje consciente, y también les haría reflexionar sobre cómo utilizan estos mecanismos atenuantes en su lengua materna, y reflexionar sobre las diferencias o similitudes que hay en los intercambios comunicativos en las dos culturas, la francesa y la española. Más precisamente, creemos que la introducción de la atenuación en la enseñanza de E/LE facilitaría un aprendizaje múltiple:

- A través del estudio del E/LE aprenderían de forma indirecta (implícita) y directa (explícita) las costumbres socioculturales, puesto que compararían el uso de los mecanismos atenuadores en ambas lenguas, el francés y el español. Esta comparación también les permitiría reflexionar sobre las diferencias en el uso de estos elementos en las dos lenguas y, así, aprenderían a utilizarlos de forma consciente. 
- Los estudiantes también aprenderían cuáles son los diferentes mecanismos atenuantes (tácticas de atenuación), y cómo pueden utilizarlos posteriormente en los diferentes contextos comunicativos en los que se desenvuelven. De este modo, los aprendices serían capaces de rechazar, negociar, llegar a compromisos o a acuerdos (siempre y cuando la situación lo necesite), salvaguardando su imagen o la de sus interlocutores, tal y como lo hacen los nativos.

\section{4. ¿CÓMO INCORPORAR LA ATENUACIÓN EN E/LE?}

Cuando se enseña pragmática en el aula de E/LE no se debería hacer de forma aislada, sino que es necesario ofrecer a los estudiantes una visión completa de los diferentes contextos y situaciones en las que podrían encontrarse, y en los que podrían utilizar las diferentes tácticas atenuantes. Para ello, como se ha demostrado en un trabajo previo (Secchi 2014), es necesario incluir los corpus discursivos orales en la enseñanza de E/LE. En este trabajo previo se han realizado dos tipos de tareas: en la primera, se han analizado los diálogos y conversaciones empleadas generalmente en los manuales de E/LE; y en la segunda, se han recogido unas muestras de corpus de conversaciones orales para destacar las ventajas en la didáctica del español como lengua extranjera (E/LE). Además, se quiso destacar la importancia de explotar fragmentos discursivos extraídos de corpus discursivos como el corpus Val.Es.Co. (2002) y C-ORAL-ROM (2005), con el objetivo de ofrecer una visión mayor -y más realista- de los aspectos del habla cotidiana que no suelen estar presentes en los manuales de E/LE. Asimismo, a través de este estudio se quiso insistir en la importancia de los corpus orales como herramienta didáctica que permita a los estudiantes trabajar con diálogos reales y, gracias a ello, adquirir las competencias discursivas que les facilitarán desenvolverse en conversaciones auténticas, y así lograr comunicarse auténticamente, esto es, como los propios nativos.

De esta forma, los estudiantes podrían entender mejor los fenómenos y los elementos atenuantes porque tendrían la posibilidad de enfrentarse a algunos casos concretos puestos en práctica, por ejemplo, a través de juegos de rol. Con esto, se les facilitaría la asimilación de los elementos atenuantes, y esto les permitiría, posteriormente, ser capaces de elegir la mejor táctica atenuante en función de las necesidades, de la situación comunicativa real en la que se encuentran y en los intercambios con nativos. Es por ello que consideramos que la introducción de la atenuación en el diseño de las actividades didácticas, y el desarrollo de las mismas en las clases de E/LE, facilitaría el conseguimiento de buenos resultados a través de un aprendizaje explícito y consciente, que solamente se puede alcanzar a través del uso de corpus orales reales en el aula.

De acuerdo con el autor Calvet (2004: 62), entendemos que es indiscutible el hecho de que "no hay lenguas sin hablantes", afirmación que legitima la introducción de los corpus orales en la enseñanza de lenguas extranjeras. Consideramos que los corpus representan una herramienta muy valiosa, tanto en el ámbito de la investigación como de la enseñanza de lenguas extranjeras (LE) y que, de acuerdo con Briz (2005: 7-12), un corpus representa "el banco de pruebas más eficaz y natural para analizar el lenguaje y más aún la actuación lingüística".

Es por ello que la mejor manera de incorporar la atenuación en E/LE es mediante el uso de un corpus compuesto por géneros discursivos dialógicos, que constituye el mejor 
formato para el desarrollo de la competencia pragmática, conversacional y atenuadora de los aprendientes. De acuerdo con Albelda (2008), los géneros discursivos dialógicos representan una herramienta que permite contextualizar las formas y las estructuras lingüísticas de cada situación comunicativa concreta y esto permite identificar cuáles son los factores situacionales que influyen en los intercambios comunicativos.

Así pues, surge la necesidad de explicitar, adaptar y guiar al estudiante para lograr un aprendizaje que favorezca el desarrollo de las habilidades comunicativas y, más específicamente, de las estrategias de atenuación a través del uso de corpus discursivos como material de producción y comprensión oral. Gracias a ello, los estudiantes pueden aprender de forma más eficaz los mecanismos que regulan los intercambios comunicativos, entender mejor los enunciados y aprender a desenvolverse de la mejor manera posible en las interacciones comunicativas reales.

\section{METODOLOGÍA Y DESCRIPCIÓN DEL CORPUS}

A pesar de que un corpus de conversaciones coloquiales reales es el que mejor se presta para la adaptación en las clases de lengua extranjera, nuestro estudio se centra en un corpus propio, constituido por intercambios lingüísticos del género debate y conversación argumentativa. La perspectiva de análisis de nuestro corpus es diferente si la comparamos con el análisis de un corpus coloquial con nativos, ya que, en nuestro caso, los informantes no son nativos españoles, sino estudiantes universitarios franceses de E/LE. No obstante, según Gallardo (1998), en ambos casos, se pueden identificar las similitudes o diferencias en cuanto a las variables sociolingüísticas (relación entre los participantes, situación comunicativa, temática), o de naturaleza pragmática (toma de turno, duración de la intervención y número de hablantes). Estas variables son también las que influyen en la elección del código lingüístico de los participantes y en los diferentes registros de habla, esto es, el registro formal, informal y coloquial. Para alcanzar dicho objetivo nos hemos propuesto realizar un estudio empírico en el que se analizará un corpus discursivo real, creado a partir de situaciones reales en diferentes contextos: formales, semiformales e informales. De acuerdo con Albelda (2004), el estudio de una categoría pragmática, como es el caso del presente estudio sobre la atenuación, debe basarse en el análisis de muestras lingüísticas reales y es por ello que hemos considerado necesario crear un corpus real como instrumento para el análisis de estos fenómenos.

Para llevar a cabo nuestra primera parte de investigación, hemos establecido basarnos en un riguroso análisis cualitativo y cuantitativo de debates y conversaciones argumentativas, con el objetivo de desarrollar un estudio empírico que permita comprobar la presencia/ ausencia de elementos atenuantes en las interacciones analizadas.

El corpus ha sido diseñado y recogido en el año 2015 en NEOMA Business School's Master Grande École de Reims (Francia), expresamente para esta investigación. El corpus lo constituyen un total de 23 grabaciones (que suman más de cuatro horas de grabación), de debates y conversaciones argumentativas entre estudiantes de máster que estudian E/LE y que tienen nivel entre un B1-C1 (de acuerdo con el Marco Común Europeo de Referencia para las lenguas, publicado en 2001). Las temáticas tratadas han sido elegidas por parte de los estudiantes entre un amplio abanico de argumentos que se les ha ofrecido. Una vez terminado el proceso de grabación, se ha procedido con la fase de transcripción del corpus. 
Para ello, se ha decidido llevar a cabo el análisis siguiendo la ficha de transcripción del grupo Val.Es.Co. (Briz y Grupo Val.Es.Co., 2002), excluyendo algunos signos de transcripción no pertinentes para nuestro estudio.

Para la transcripción del corpus propio hemos decidido emplear solo una parte de los signos de transcripción creados por el grupo Val.Es.Co., puesto que en nuestro estudio dejaremos de lado algunas de las funciones prosódicas para centrarnos exclusivamente en las que pueden influir en la clasificación de los elementos atenuantes presentes en las interacciones grabadas. Albelda (2005: 381-388) define el método de transcripción Val. Es.Co. como un sistema lo suficientemente estrecho para permitir que el lector pueda reproducir de forma fiel la conversación original sin que los signos de transcripción dificulten la lectura.

Para el análisis del corpus propio se ha operado desde una perspectiva cualitativa y cuantitativa. Para el análisis cualitativo, se ha seguido la ficha metodológica de Albelda et alii (2014), en la que se establece una nómina de formas atenuantes (que se ha cruzado con la nómina del PCIC 2006) y una serie de variables pragmáticas y situacionales que permiten reconocer la función atenuante. Gran parte de estas variables han sido extraídas de la mencionada ficha, mientras que otras han sido añadidas teniendo en cuenta la especificidad del grupo analizado y del contexto. Para el análisis cuantitativo, se han identificado y extraído los resultados de los mecanismos de atenuación de acuerdo con su clasificación lingüística y su particular función atenuante.

A través de este corpus, lo que se pretende observar y analizar es cómo los informantes grabados utilizan, de manera consciente o inconsciente, la atenuación, cuándo la utilizan y de qué forma, y si hay intercambios en los que deberían usarla pero no la usan.

Gracias a este estudio, no solo se pretende interpretar los datos referidos al conocimiento y a las capacidades de estos estudiantes de utilizar la atenuación en los intercambios comunicativos, sino también sus posibles carencias o su falta de habilidad pragmática en el contexto comunicativo.

\section{DESCRIPCIÓN DE LA FICHA DE ANÁLISIS ES.VAR.ATENUACIÓN}

Para identificar y analizar los elementos de atenuación existentes en nuestros corpus hemos decidido diseñar una nueva ficha de análisis apoyándonos en la ficha ES.VAR.ATENUACIÓN (Albelda et alii 2014, Villalba 2015), que fue diseñada para cuantificar datos y llevar a cabo análisis contrastivos de la atenuación en corpus discursivos de distintas variedades del español.

A partir de esta ficha ya existente hemos decidido utilizar las variables que mejor se adaptan a nuestro estudio y, de acuerdo con nuestros objetivos, hemos decidido incluir nuevas funciones y variables de análisis. Es preciso afirmar que, hasta el momento, la ficha ES.VAR.ATANUACIÓN ha sido utilizada exclusivamente para el análisis de conversaciones y entrevistas semidirigidas del corpus Val.Es.Co. (Briz et alii 2002), y también para el análisis de juicios orales (Villalba 2015), con buenos resultados.

A continuación, se incluye una tabla a modo de esquema (Tabla 1: ficha ES.VAR.ATENUACIÓN) en la que se resumen sintéticamente los factores de análisis y las variables que constituyen la ficha ES.VAR.ATENUACIÓN original a la que posteriormente se le han añadido nuevos elementos de análisis que se detallarán más adelante en el epígrafe 7.1. 


\begin{tabular}{|c|c|}
\hline $\begin{array}{l}\text { Bloques de los } \\
\text { factores de análisis }\end{array}$ & Variables de análisis \\
\hline $\begin{array}{l}\text { (A) Función de la } \\
\text { atenuación }\end{array}$ & 1. Función general del atenuante \\
\hline $\begin{array}{l}\text { (B) Procedimiento } \\
\text { de atenuación }\end{array}$ & $\begin{array}{l}\text { 2. Tácticas lingüísticas y no lingüísticas de atenuación } \\
\text { 2.1. Modificadores morfológicos internos: sufijos (diminutivos). } \\
\text { 2.2. Modificadores externos: cuantificadores minimizadores, } \\
\text { aproximativos o difusores significativos proposicionales o } \\
\text { extraproposicionales. } \\
\text { 2.3. Términos o expresiones más suaves en el contenido significativo. } \\
\text { 2.4. Empleo de palabras extranjeras. } \\
\text { 2.5. Usos modalizadores (“dislocados”) de los tiempos verbales. } \\
\text { 2.6. Empleo de verbos, construcciones verbales, así como de partículas } \\
\text { discursivas con valor modal que expresan opiniones en forma de } \\
\text { duda o de probabilidad. } \\
\text { 2.7. Empleo de verbos, construcciones verbales y partículas discursivas } \\
\text { que expresan fingimiento de incertidumbre, de incompetencia o de } \\
\text { ignorancia. } \\
\text { 2.8. Construcciones acotadoras de la opinión. } \\
\text { 2.9. Peticiones, preguntas, mandatos u órdenes expresados de forma } \\
\text { indirecta. } \\
\text { 2.10. Expresiones de disculpa. } \\
\text { 2.11. Estructuras sintácticas o significados condicionales, concesivos o } \\
\text { temporales. } \\
\text { 2.12. Partículas y construcciones justificadoras o de excusa. } \\
\text { 2.13. Elipsis de la conclusión, estructuras suspendidas o estructuras } \\
\text { truncadas. } \\
\text { 2.14. Impersonalizaciones (I). } \\
\text { 2.15. Impersonalizaciones (II) a través del recurso al estilo directo. } \\
\text { 2.16. Movimiento de reformulación. } \\
\text { 2.17. Concesividad. } \\
\text { 2.18. Partículas discursivas (sobre todo, evidenciales). } \\
\text { 2.19. Partículas discursivas y expresiones de control de contacto. } \\
\text { 2.20. Uso de formas de tratamiento y de fórmulas apelativas } \\
\text { convencionalizadas. } \\
\text { 2.21. Empleo de elementos PROSóDICOS, paralingüísticos o gestuales. } \\
\text { 2.22. Otros. } \\
\text { 3. Número de procedimientos de atenuación }\end{array}$ \\
\hline $\begin{array}{l}\text { (C) Factores } \\
\text { estructurales }\end{array}$ & $\begin{array}{l}\text { 4. Posición discursiva del atenuante en el miembro discursivo al que afecta } \\
\text { 5. Tipología textual }\end{array}$ \\
\hline $\begin{array}{l}\text { (D) Factores } \\
\text { enunciativos }\end{array}$ & $\begin{array}{l}\text { 6. Contenido de lo dicho en relación con las imágenes de las personas } \\
\text { 7. Fuerza ilocutiva del acto de habla }\end{array}$ \\
\hline $\begin{array}{l}\text { (E) Factores } \\
\text { situacionales }\end{array}$ & $\begin{array}{l}\text { 8. Temática } \\
\text { 9. Fin de la interacción }\end{array}$ \\
\hline
\end{tabular}

Tabla 1. Ficha Es.Var.Atenuación (Albelda, Briz, Cestero, Kotwica y Villalba 2014). 


\section{ELEMENTOS INCLUIDOS EN LA NUEVA FICHA DE ANÁLISIS Y EN LA TA- BLA DE EXCEL}

En la primera fase de nuestro trabajo hemos llevado a cabo una prueba de aplicación de la ficha ES.VAR.ATENUACIÓN original y, puesto que en nuestro estudio analizamos géneros discursivos diferentes de los que han sido analizados con la ficha ES.VAR.ATENUACIÓN, esto es, debates y conversaciones argumentativas llevadas a cabo por participantes no hispanohablantes, hemos procedido a la modificación de la misma. Estas diferencias han representado una limitación de la aplicabilidad de la ficha original y, consecuentemente, justifican la inclusión de algunas modificaciones de la ficha original y las nuevas aportaciones para el análisis de la presencia/ausencia de la atenuación en los géneros discursivos analizados y que constituyen nuestro corpus propio.

En este apartado se detallan todos los elementos que constituyen nuestra tabla Excel incluyendo tanto las funciones y variables extraídas de la ficha ES.VAR.ATENUACIÓN original (Albelda et alii 2014), como los nuevos elementos incluidos en la ficha de análisis original y que constituyen nuestra aportación.

\subsection{Nuevas aportaciones}

La creación de tres nuevas categorías de análisis constituye nuestra aportación, e implementación, a la ficha de análisis ES.VAR.ATENUACIÓN. Más concretamente, a través de estas tres nuevas categorías de análisis se pretende identificar los siguientes elementos:

- Presencia o ausencia de un mecanismo atenuante

- Juicio pragmático sobre el uso del atenuante

- Intervenciones que pueden dañar la imagen del interlocutor

Cada una de estas tres categorías de análisis se detallará más adelante en el epígrafe 7.2, donde también se detallarán las variables que se incluyen en estas tres nuevas categorías.

Como se ha dicho anteriormente, nuestra ficha de análisis es el resultado de la adaptación de la ficha ES.VAR.ATENUACIÓN. A pesar de que algunos de los objetivos del análisis siguen siendo los mismos, es decir, identificar las funciones atenuadoras y los procedimientos lingüísticos atenuadores (Albeda et alii 2014), nuestra aportación pretende añadir nuevos objetivos, como el de analizar las interacciones identificando la ausencia de atenuación y también identificar el juicio pragmático sobre el uso del atenuante sin olvidar el contexto en el que se han llevado a cabo las interacciones y el bagaje lingüístico de los discentes en E/LE.

Para el análisis de nuestro corpus hemos decidido volcar los elementos que constituyen nuestra nueva ficha de análisis en el diseño de una tabla en Excel. Los elementos de nuestra ficha de análisis han sido repartidos en veinticinco columnas (desde la columna A hasta la columna Y). En lo que sigue detallaremos cuáles han sido los elementos que hemos incluido en nuestra ficha de análisis y también en qué orden han sido colocados en la tabla de análisis en Excel. Además, se indicarán tanto los elementos extraídos de la ficha ES.VAR. ATENUACIÓN, útiles para nuestro estudio, como los nuevos elementos que hemos creado especificadamente para nuestro estudio y que constituyen nuestra aportación a la ficha.

A continuación, detallaremos cuáles son los elementos que hemos elegido y en qué orden han sido colocados en el diseño de nuestra tabla de análisis en Excel. 


\subsection{Bloques de los factores de análisis y diseño de la tabla de clasificación}

Para la individualización de la atenuación en las transcripciones se han considerado tanto los elementos lingüísticos, como los elementos no lingüísticos que nos han permitido descodificar la atenuación e identificar en los fragmentos analizados las tácticas y elementos atenuantes utilizados por el hablante.

Para ello nos hemos atenido a la clasificación de los autores Briz y Albelda (2013), que dividen las tácticas atenuantes en dos grupos: las tácticas que buscan la ocultación de la información y las tácticas que buscan la relativización o indeterminación de la información (Briz y Albelda 2013: 303).

Para el análisis de cada transcripción de la grabación, sea un debate o una conversación argumentativa, se ha empleado una tabla diferente (diseñada en una hoja de Excel). En cada tabla se han indicado las líneas de referencia a la transcripción de cada debate o conversación argumentativa y también se han incluido los fragmentos analizados donde se ha encontrado atenuación o falta de atenuación o donde el contexto comunicativo nos indica que sería mejor que el intercambio comunicativo sea atenuado, para evitar un posible daño a la imagen propia o a la del interlocutor.

Para el análisis de cada transcripción, tanto de debates o de conversaciones argumentativas, nos hemos servido de distintas hojas Excel. Cada hoja Excel incluye todos los elementos de análisis de nuestra ficha y todos los fragmentos analizados por cada transcripción que constituye nuestro corpus propio.

Todos los elementos de análisis con múltiples variables se contabilizarán en un recuento al final en cada hoja de análisis de Excel. Esto nos ha permitido establecer con exactitud el número de funciones y variables encontradas.

\section{$N^{o}$ de línea}

En las casillas de la columna A de la tabla Excel se ha incluido el número de la línea de la transcripción, del debate o de la conversación argumentativa, analizada.

\section{Fragmento}

En las casillas de la columna B de Excel se ha incluido el fragmento donde hemos encontrado el/los elemento/os o la construcción atenuante.

Recordamos que en nuestro estudio para el análisis de las interacciones se ha tomado como unidad de análisis el acto de habla (Searle 1969), que representa la unidad más pequeña de la comunicación que realiza una acción y que tiene sentido completo.

Se han marcado en rojo los fragmentos que han sido identificados como elementos atenuados y, de lo contrario, se han marcado en azul los fragmentos que han sido identificados como elementos no atenuados pero que sí deberían ser atenuados para evitar un posible daño a la imagen propia o a la del interlocutor.

Todos los fragmentos han pasado por un análisis de funciones y variables. Para algunas funciones, las variables que se pueden elegir son la 0 (siendo 0 el número que utilizaremos para indicar que una específica variable no está presente en esa función), o la 1, 2, 3, 4 y 5 que hemos usado para indicar la presencia de una determinada variable. Este sistema de 
clasificación nos ha permitido contabilizar por cada transcripción analizada (al final de cada tabla Excel), el total de las funciones y variables que hemos encontrado. Posteriormente, gracias a los datos obtenidos hemos podido proceder a la creación de gráficos específicos que nos han permitido extraer conclusiones en cuanto a la presencia, a la ausencia, a la carencia o al uso inadecuado de la atenuación en las conversaciones argumentativas y en los debates analizados.

\subsubsection{Presencia o ausencia de un mecanismo atenuante}

Para establecer si los elementos analizados tienen, o no, función atenuante es oportuno analizar las transcripciones de las interacciones que se pretenden estudiar teniendo en cuenta algunos criterios que las autoras Albelda (2014b) y Villalba (2012), clasifican en tres puntos:

1) Contexto interactivo concreto: se analiza la situación comunicativa en la que ha habido atenuación. Puesto que el mecanismo atenuante es un mecanismo estratégico, esto es, una estrategia comunicativa, el motivo por el cual se ha usado se puede identificar.

2) Criterio de ausencia: la ausencia o la eliminación del elemento atenuante supondría cambios en la fuerza ilocutiva de los enunciados.

3) Criterio de solidaridad: la atenuación no suele aparecer de manera aislada, esto significa que la presencia de otros elementos atenuantes en el fragmento analizado es otro factor que nos guía a la hora de identificar la presencia de una función atenuante.

Tras haber tenido en cuenta estos elementos, y también a los discentes que pretendemos analizar en nuestro estudio (estudiantes universitarios franceses), hemos plasmado la necesidad de crear tres nuevos elementos de clasificación, uno que nos facilite identificar la presencia o ausencia de un mecanismo atenuante, otro que nos permita atribuir un juicio pragmático sobre el uso del atenuante y un último elemento que nos permita clasificar las intervenciones que pueden dañar la imagen del interlocutor.

El primer nuevo elemento de clasificación (presencia o ausencia de un mecanismo atenuante), incluido en la ficha de análisis, nos permite identificar no solamente cuando hay atenuación sino también cuando no la hay pero sí debería haberla porque el contexto comunicativo lo requiere. Por último, esta función también nos permite identificar cuando hay atenuación pero no debería haberla porque en la situación comunicativa concreta no es necesaria.

Para la clasificación de nuestros fragmentos en las casillas de la columna C se tendrá que marcar la presencia o ausencia de un mecanismo atenuante y, para ello, se tendrá que elegir entre una de las cuatro variables:

- Hay

- No hay pero debería haber (motivo: falta de vocabulario o de conocimiento de la lengua)

- No hay pero debería haber (motivo: falta de habilidad pragmática en el contexto)

- ay pero no debería haber 


\section{Variable 1: Hay atenuación}

Esta variable indica la presencia de atenuación en el fragmento analizado, sean elementos atenuantes concretos o circunloquios, que el hablante utiliza en su interacción para lograr sus objetivos y que pueden estar de acuerdo o en desacuerdo con su interlocutor (Briz, 2003; 2007). En el caso de que se encuentren elementos atenuantes, se indicará en la celda de la función correspondiente la relativa variable, en este caso 1 (hay atenuación).

Variable 2: No hay atenuación pero debería haberla (motivo: falta de vocabulario o de conocimiento de la lengua)

El hablante no sabe, o no consigue, expresar sus opiniones por falta de léxico o de conocimiento de los elementos atenuantes, sin que se generen conflictos; consecuentemente, aunque los intercambios comunicativos necesiten ser atenuados -en un ataque a la imagen del otro o en respuesta a un ataque a la propia imagen-, el hablante no es capaz de preservar ni su imagen, ni la imagen del otro, para poder avanzar en un intercambio constructivo y cooperativo.

Se considera que la intervención del hablante en el intercambio comunicativo requiere ser atenuado porque representa -o puede representar-, un daño a la propia imagen o a la del interlocutor.

La falta de atenuación por parte del hablante puede deberse a la falta de vocabulario o un conocimiento de la lengua no lo suficientemente adecuado para permitirle utilizar elementos atenuantes.

En cuanto a la pobreza de vocabulario, el hablante desconoce, o no es capaz de utilizar de forma adecuada los términos necesarios que podría emplear para atenuar lo dicho -a través de tácticas atenuantes-y por lo tanto, su intervención carece de atenuación a pesar de que el contexto comunicativo lo requiera.

En el caso de falta de conocimiento de la lengua, el hablante no es capaz de expresar lo que quiere decir de forma atenuada y su intervención falta de coherencia, o de cohesión, debido a que utiliza un lenguaje empobrecido y con escasa calidad léxica (hay repetición de palabras o uso de palabras con un significado extensivo), o simplemente porque su intervención carece de claridad de expresión. Por lo tanto, al no tener un adecuado conocimiento de la lengua, o del vocabulario, el hablante no es capaz de expresar lo dicho de forma clara, es decir, no posee las habilidades necesarias para poder expresarse de forma atenuada evitando así que se produzca un daño a la propia imagen o a la de su interlocutor.

En el caso de que en el fragmento analizado no hubiera atenuación pero sí debería haberla (por las razones explicadas anteriormente), se procederá a marcar en la celda de la función correspondiente, la relativa variable, en este caso 2 (no hay atenuación pero debería haberla, motivo: falta de vocabulario o de conocimiento de la lengua).

Variable 3: No hay atenuación pero debería haberla (motivo: falta de habilidad pragmática en el contexto)

El interlocutor no consigue atenuar lo dicho aunque si su intervención en la conversación requiere ser atenuada porque puede provocar un daño a la imagen del interlocutor o del yo 
del proprio hablante. En estos casos el hablante no es capaz de expresar lo dicho de forma atenuada, ni a través de elementos atenuantes, ni a través de circunloquios que consigan atenuar lo dicho. El hablante carece -o desconoce- las habilidades pragmáticas que debería aplicar en los intercambios comunicativos que lo requieran de modo que no se produzcan conflictos.

Se considera que no hay atenuación pero debería haberla (motivo: de falta de habilidad pragmática en contexto), cuando las intervenciones del hablante en el intercambio comunicativo son directas y su(s) interlocutor(es) interpreta(n), estas intervenciones como un ataque directo a la propia imagen a pesar de que no haya habido ningún ataque previo a su intervención y, por ello, no se pueda considerar como una respuesta a otro ataque. En el caso de que en el fragmento analizado no hubiera atenuación, pero sí debiera haberla (motivo: falta de habilidad pragmática en el contexto), se indicará en la celda de la función correspondiente, la variable relativa, en este caso, la 3 (no hay atenuación pero debería haberla; motivo: falta de habilidad pragmática en el contexto).

\section{Variable 4: Hay atenuación pero no debería haberla}

El hablante ha recurrido a la atenuación a pesar de que la situación comunicativa no lo requiera, por lo tanto su táctica atenuadora se considera inadecuada en el específico intercambio comunicativo. Para la identificación de esta categorización se tendrán en cuenta los siguientes elementos:

- el contexto comunicativo concreto

- el contenido de las intervenciones anteriores y posteriores entre los interlocutores que participan en el intercambio comunicativo

- las respuestas a un ataque a la imagen del interlocutor: cuando la reacción a un ataque es una respuesta que no necesita ser atenuada

En el caso de que en el fragmento analizado hubiera atenuación, pero no debería haberla, se procederá a marcar en la celda de la función correspondiente, la variable relativa, en este caso la 4 (hay atenuación per no debería haberla).

\subsubsection{Juicio pragmático sobre el uso del atenuante}

El segundo elemento nuevo que se ha incluido en la ficha de análisis es el juicio pragmático sobre el uso del atenuante. Este elemento de análisis nos permite clasificar cómo el hablante ha utilizado en sus intervenciones comunicativas los elementos atenuantes a través de unos parámetros que pretenden medir la corrección del uso de los elementos atenuantes teniendo en cuenta el contexto comunicativo específico en el que han sido utilizados.

En nuestro estudio, la clasificación del juicio pragmático sobre el uso del atenuante es de fundamental importancia, puesto que nos permite entender mejor cuáles son las dificultades de los estudiantes universitarios franceses analizados en el uso de las tácticas y de los elementos atenuantes. Esto también representaría el punto de partida para el diseño de materiales para las clases de E/LE, en el que se incluyan corpus reales como instrumento de aprendizaje y de mejora de las habilidades comunicativas de los aprendientes. 
Para la clasificación de nuestros fragmentos en las casillas de la columna D (juicio pragmático sobre el uso del atenuante), se tendrá que elegir entre una de las cuatro variables:

- No hay atenuación

- Hay atenuación pero no es adecuada a la situación comunicativa

- La atenuación es adecuada pero podría ser más adecuada

- La atenuación es totalmente adecuada a la situación comunicativa

\section{Variable 0: No hay atenuación}

Esta variable indica que en el fragmento analizado no hay atenuación. Esto significa que siempre y cuando en la columna C (Presencia o ausencia de mecanismos atenuante) se haya clasificado la variables 2 (No hay atenuación pero debería haberla; motivo: falta de vocabulario o de conocimiento de la lengua), o la variable 3 (No hay atenuación pero debería haberla; motivo: falta de habilidad pragmática en el contexto), la variable de la columna D (Juicio pragmático sobre el uso del atenuante) siempre tendrá que ser la variable 0 , es decir, No hay atenuación.

\section{Variable 1: Hay atenuación pero no es adecuada a la situación comunicativa}

Esta variable indica que en el fragmento analizado hay atenuación pero que no es adecuada a la situación comunicativa. Puesto que la clasificación de esta variable puede parecer difícil de identificar, o tendencialmente arbitraria, a continuación presentamos, a modo de ejemplo, un caso en el que un elemento atenuante no se considera adecuado a la situación comunicativa. Sirva de muestra el ejemplo 1:

(1) C: Estados Unidos publicó/ un informe// en mil novecientos noventa y ocho afirmando que Cuba no representa una amenaza poor- militar/ significativa por los Estados Unidos/ y por otros países de la región/// así yo pienso quee $\rightarrow$ que el embargo noo- noo es una buena cosas

B: sí/ estoy de acuerdo coon- con usted/ $\mathrm{Y}$ al mismo tiempo estoy- no estoy de acuerdo con usted/ [porque]

C:

[(RISAS)]

Como se observa en el ejemplo arriba indicado (en negrita), el hablante B contesta al hablante $\mathrm{C}$ intentando atenuar su disconformidad, pero su táctica atenuadora es poco clara e/o ineficaz, de hecho la respuesta de B provoca que el interlocutor $\mathrm{C}$ en lugar de contestarle se ríe, debido a que la intervención de $\mathrm{B}$ es confusa e incoherente. En este intercambio podemos deducir que $\mathrm{B}$, a pesar de no estar de acuerdo con $\mathrm{C}$, no quiere contestarle diciéndole que no tiene la razón, puesto que esto supondría un ataque a la imagen de su interlocutor (C). Tras el análisis del intercambio comunicativo en contexto, podemos identificar que B, a pesar de que no lo consiga, intenta utilizar una táctica atenuadora de concesividad -concretamente un movimiento concesivo-opositivo- que minimice su disconformidad.

En todos los casos como éste, y en otros casos semejantes, se considerarán $-\mathrm{y}$ clasificarán- como elementos atenuadores inadecuados a la situación comunicativa todas las intervenciones en las que los hablantes no hayan conseguido utilizar de manera clara los ele- 
mentos, o tácticas, atenuantes pero que, a pesar de ello, en el análisis de los intercambios comunicativos se pueda entender la finalidad atenuadora que el hablante hubiera querido lograr.

\section{Variable 2: La atenuación es adecuada pero podría ser más adecuada}

Se establece que la atenuación es adecuada, pero podría ser más adecuada al contexto comunicativo concreto cuando el hablante recurre constantemente al uso de los mismos elementos atenuadores, o a los mismos circunloquios, para mitigar la fuerza ilocutiva del posible acto amenazador de su enunciado. En este sentido se considera que el hablante tiene un conocimiento limitado, o no es capaz de utilizar de la mejor forma otras tácticas u otros elementos atenuantes, diferentes de los que ya ha utilizado anteriormente en otras intervenciones dentro de los mismos intercambios comunicativos analizados.

Consecuentemente se puede apreciar que la elección de las tácticas atenuadoras por parte del hablante no haya sido tan eficaz como el hablante hubiese querido y que, al repetirse sin diversificar, la eficacia de sus tácticas no sea lo más adecuado a lo que la situación comunicativa requiere en ese determinado contexto.

\section{Variable 3: La atenuación es totalmente adecuada a la situación comunicativa}

Esta variable indica que en el fragmento analizado hay atenuación y que su uso en el contexto es totalmente adecuado a la situación comunicativa y en el intercambio analizado por cuanto responde a los criterios de claridad, validez y pertinencia.

\subsubsection{Función general del atenuante}

El hablante recurre al uso de la atenuación en dos casos, bien para protegerse a sí mismo o bien para evitar perjudicar la relación con el otro (Holmes 1984, Caffi 1999), es decir, el hablante usa la atenuación para evitar provocar un daño a la imagen de su interlocutor o del yo del propio hablante.

En cuanto a las funciones específicas de la atenuación en cuanto a imagen Briz (2011: 3-21) las clasifica en tres tipos:

- la función autoprotectora (de autoprotección)

- la función preventiva (de prevención)

- la función reparadora (de curación o reparación)

Variable 1: La función de autoprotección

Se produce cuando el hablante utiliza mecanismos atenuadores con el objetivo de proteger la imagen de sí mismo.

\section{Variable 2: La función de prevención}

Se produce cuando el hablante anticipa el efecto que podría tener lo dicho (un acto amenazante) en su interlocutor y para ello recure al uso de la atenuación para proteger la imagen de su interlocutor. 


\section{Variable 3: La función de curación o reparación}

Se produce cuando en la interacción comunicativa ha habido una amenaza, o un daño a la imagen de un interlocutor, y el hablante suaviza o repara los efectos negativos que han provocado el daño a la imagen.

\subsubsection{Tácticas lingüisticas y no lingüisticas de atenuación}

De las veintidós tácticas lingüísticas y no lingüísticas de atenuación que constituyen la ficha ES.VAR.ATENUACIÓN, hemos decidido extraer únicamente diecisiete tácticas de análisis, que son las que nos han permitido llevar a cabo la primera parte de análisis de nuestro trabajo y que se detallan continuación:

\subsubsection{Modificadores morfológicos internos: sufijos (diminutivos)}

Variable 0: No modificadores morfológicos internos: sufijos (diminutivos).

Variable 1: Modificadores morfológicos internos: sufijos (diminutivos).

7.2.4.2. Modificadores externos: cuantificadores minimizadores, aproximativos o difusores significativos proposicionales o extraproposicionales

Variable 0: No modificación morfológica externa

Variable 1: Modificadores externos: cuantificadores minimizadores, aproximativos o difusores significativos proposicionales o extraproposicionales.

\subsubsection{Empleo de palabras extranjeras}

Variable 0: No empleo de palabras extranjeras

Variable 1: Empleo de palabras extranjeras

\subsubsection{Usos modalizadores (“dislocados”) de los tiempos verbales}

Variable 0: No usos modales (dislocados) de los tiempos verbales.

Variable 1: Usos modales del tiempo verbal

7.2.4.5. Empleo de verbos, construcciones verbales, así como de partículas discursivas con valor modal que expresan opiniones en forma de duda o de probabilidad

Variable 0: No verbos, partículas y construcciones verbales que expresan las opiniones en forma de duda o probabilidad

Variable 1: Verbos, partículas y construcciones verbales que expresan las opiniones en forma de duda o probabilidad

7.2.4.6. Empleo de verbos, construcciones verbales y partículas discursivas que expresan fingimiento de incertidumbre, de incompetencia o de ignorancia

Variable 0: No verbos, partículas discursivas y construcciones verbales que expresan fingimiento de incertidumbre, de incompetencia o de ignorancia 
Variable 1: Verbos, partículas discursivas y construcciones verbales que expresan fingimiento de incertidumbre, de incompetencia o de ignorancia

\subsubsection{Construcciones acotadoras de la opinión}

Variable 0: No construcciones acotadoras de la opinión

Variable 1: Construcciones acotadoras de la opinión

7.2.4.8. Peticiones, preguntas, mandatos u órdenes expresados de forma indirecta

Variable 0: No peticiones, preguntas, mandatos expresados de forma indirecta con una formulación afirmativa o negativa

Variable 1: Peticiones, preguntas, mandatos expresados de forma indirecta con una formulación afirmativa o negativa

\subsubsection{Expresiones de disculpa}

Variable 0: No expresiones de disculpa

Variable 1: Expresiones de disculpa

7.2.4.10. Partículas y construcciones justificadoras o de excusa

Variable 0: No partículas y construcciones justificadoras o de excusa

Variable 1: Partículas y construcciones justificadoras o de excusa

\subsubsection{Impersonalizaciones (I)}

Variable 0: No impersonalizaciones (I)

Variable 1: Apelar al juicio de la mayoría o a un interlocutor general mediante pronombres (se, uno, tú, nosotros inclusivo)

Variable 2: Apelar al juicio de la mayoría mediante formas verbales impersonales, formas de despersonalización del origen deíctico del enunciado (por lo que dicen, según cuentan, por lo visto, al parecer, según parece)

Variable 3: Apelar a la institución o entidad que se representa

Variable 4: Encubrir la opinión propia en la opinión de otras personas, o en la autoridad de alguien o de algo

Variable 5: Generalizar para despersonalizar

\subsubsection{Impersonalizaciones (II) a través del recurso al estilo directo}

Variable 0: No impersonalizaciones (II)

Variable 1: Citar las palabras o el pensamiento de otra persona

Variable 2: Citar lo que dice el acervo común

Variable 3: Citar las palabras o el pensamiento del propio hablante como si no fueran suyas 
7.2.4.13. Movimiento de reformulación

Variable 0: No movimiento de reformulación

Variable 1: Movimiento de reformulación

7.2.4.14. Concesividad

Variable 0: No concesividad

Variable 1: Concesividad

7.2.4.15. Partículas discursivas (sobre todo, evidenciales)

Variable 0: No partículas discursivas evidenciales

Variable 1: Partículas discursivas evidenciales

7.2.4.16. Partículas discursivas y expresiones de control de contacto

Variable 0: No partículas discursivas y expresiones de control de contacto

Variable 1: Partículas discursivas y expresiones de control de contacto

7.2.4.17. Empleo de elementos PROSÓDICOS, paralingüísticos o gestuales

Variable 0: No elementos prosódicos, paralingüísticos o gestuales

Variable 1: Elementos prosódicos, paralingüísticos o gestuales

\subsection{Número de procedimientos de atenuación}

Variable 1: Un procedimiento de atenuación

Variable 2: Dos procedimientos de atenuación

Variable 3: Tres procedimientos de atenuación

Variable 4: Cuatro procedimientos de atenuación

Variable 5: Más de cuatro procedimientos de atenuación (se indicará el número exacto)

\subsection{Intervenciones que pueden dañar la imagen del interlocutor}

El tercer elemento nuevo, que se ha añadido en la ficha de análisis, es el de las intervenciones que pueden dañar la imagen del interlocutor. Con esta función se pretende analizar y clasificar de qué forma las intervenciones de los participantes a la interacción comunicativa puede dañar, directa o indirectamente, la imagen del interlocutor y cuáles son los mecanismos que podrían desencadenar un ataque directo o indirecto.

Variable 0: No hay atenuación

Esta variable indica que en el fragmento analizado no hay atenuación. Esto significa que siempre y cuando en la columna C (Presencia o ausencia de mecanismos atenuantes) 
se haya clasificado la variable 2 (No hay atenuación pero debería haberla; motivo: falta de vocabulario o de conocimiento de la lengua), o la variable 3 (No hay atenuación pero debería haberla; motivo: falta de habilidad pragmática en el contexto), la variable de la columna X (Intervenciones que pueden dañar la imagen del interlocutor) siempre tendrá que ser la variable 0, es decir, No hay atenuación.

Variable 1: Provocación y ataque

Esta variable se seleccionará en todos los casos en que se encuentre un elemento en una intervención en la que el hablante ataca expresamente su interlocutor provocándolo. Como consecuencia, el hablante causa un daño a la imagen de su interlocutor, que probablemente reaccionará con otro ataque.

Variable 2: Reacción a un ataque

Esta variable se seleccionará en todos los casos en que se encuentre un elemento, en una intervención, en la que el hablante se defiende a un ataque o provocación, contraatacando.

Variable 3: Se expresa un contenido que ataca la imagen indirectamente

Esta variable se seleccionará en todos los casos en que se encuentre un elemento, en una intervención, en la que el hablante provoca un ataque sin quererlo. Podemos reconocer esta variable porque el hablante, en el intercambio comunicativo, no ataca directamente a su interlocutor pero tampoco utiliza elementos atenuantes que lo dicho.

\subsection{Incidencias, observaciones, comentarios}

Se ha incluido una sección para las incidencias, para los casos de mecanismos atenuantes que no corresponden a ninguna de las clasificaciones presentes en la ficha y se comenta su función.

\section{CONCLUSIONES}

En este trabajo se pretende mostrar los pasos que hemos dado en el análisis de una parte de nuestro corpus oral propio (nueve de las veintitrés transcripciones que constituyen el corpus completo), para la identificación y clasificación de los elementos -y tácticas- atenuantes utilizadas por estudiantes universitarios franceses de E/LE. Para ello, se ha utilizado y adaptado la ficha de análisis de la atenuación del grupo ES.VAR.ATENUACIÓN, aplicándola al género analizado: el debate y la conversación argumentativa.

Tras una descripción del corpus oral, se han descrito las funciones y las variables útiles para nuestro estudio, que han sido extraídas de la ficha ES.VAR.ATENUACIÓN. La aplicación de la ficha al corpus analizado ha generado la necesidad de crear nuevos elementos de análisis que han sido implementados a la ficha y que constituyen nuestra aportación. Hay que subrayar que estas nuevas aportaciones a la ficha han sido una necesidad, no solo debido al género concreto objeto de análisis (el debate y la conversación argumentativa), sino sobre 
todo por el tipo de hablantes analizados, aprendiente de E/LE. Esta implementación nos ha permitido identificar y clasificar tres nuevas variantes, como la presencia o ausencia de un mecanismo atenuante, el juicio pragmático sobre el uso del atenuante y las intervenciones que pueden dañar la imagen del interlocutor.

Gracias a la aplicabilidad de la ficha ES.VAR.ATENUACIÓN y su adaptabilidad, ha sido posible la creación de un modelo de análisis para la identificación de la presencia/ausencia de atenuación en un corpus de adquisición del español de estudiantes universitarios franceses de E/LE.

Finalmente, la creación de un modelo de análisis para la identificación de la presencia/ ausencia de atenuación en un corpus de adquisición del español de estudiantes franceses, nos permitirá extraer conclusiones en cuanto al conocimiento, las capacidades y carencias en el uso de mecanismos atenuantes en el grupo de estudiantes franceses de E/LE analizados en esta primera porción del corpus, y que el mismo pueda servir de punto de partida para futuras investigaciones.

\section{Referencias bibliográficas}

Albelda, M. (2004). "Cortesía en diferentes situaciones comunicativas. La conversación coloquial y la entrevista sociológica semiformal". Pragmática sociocultural: estudios sobre el discurso de cortesía en español. Barcelona: Ariel, pp. 109-369.

Albelda, M. (2005). "Sistemas de transcripción de los corpus orales del español". Perspectivas interdisciplinares de la lingüística aplicada. Asociación Española de Lingüística Aplicada, AESLA, pp. 381-388.

Abelda, M. (2008). La enseñanza de la conversación coloquial. Madrid: Arco Libros.

Albelda, M. y A. Briz (2010). “Aspectos pragmáticos. Cortesía y atenuantes verbales en las dos orillas a través de muestras orales”. En Izquierdo, M. y J. Enguita (Eds.), La lengua española en América: Normas y usos actuales, pp. 237-260: http://www.uv.es/aleza/esp.am.pdf (10-06-2017).

Albelda, M., Briz, A., Cestero, A. M., Kotwica, D. y C. Villalba (2014). "Ficha metodológica para el análisis pragmático de la atenuación en corpus discursivos del español. (ES.POR.ATENUACIÓN)", Oralia, 17, pp. 7-62.

Albelda, M., Briz, A., Cabedo, A., Estellés, M., González, V., Hidalgo, A., Llopis, A., Padilla, X., Pérez, M., Pons, S., Ruiz, L., Sanmartín, J., Montañez, M. P., Kotwica, D., Villalba, C., LópezNavarro, E., Company, S., Pascual, E., Salameh, S., Soler, S. y Uclés, G. (2014). "Las unidades del discurso oral. La propuesta Val. Es. Co. de segmentación de la conversación (coloquial)”. Estudios de Lingüística Española, 35, pp. 13-73.

Bazzanella, C., Caffi, C. y M. Sbisà (1991). "Scalar dimensions of illocutionary force", Speech acts: fiction or reality, pp. 63-76.

Briz, A. y Grupo Val.Es.Co. (2002). Corpus de conversaciones coloquiales. Madrid: Arco Libros.

Briz, A. (2003). "La estrategia atenuadora en la conversación cotidiana española". En Bravo, D. (ed.), Actas del Primer Coloquio del Programa Edice. La perspectiva no etnocentrista de la cortesía: identidad sociocultural de las comunidades hispanohablantes. Estocolmo: Universidad de Estocolmo, pp. 17-46.

Briz, A. (2005). "Los corpus del español hablado: presentación”, Oralia, 8, pp. 7-12.

Briz, A. (2006), "Unidades del discurso, partículas discursivas y atenuantes. El caso de "no/ tienes razón”. En Falk, J., Gille, J. y Wachtmeister, F. (coords.), Discurso, Interacción e identidad. Homenaje a Lars Fant. Estocolmo: Universidad de Estocolmo, pp. 13-36.

Briz, A. (2007). "Para un análisis semántico, pragmático y sociopragmático de la cortesía atenuadora en España y América", Lingüistica Española Actual, 29/1, pp. 5-40. 
Briz, A. y Universidad de Estudios Extranjeros de Beijing (2011). "La atenuación como categoría pragmática", Actas del VII Congreso Internacional de la Asociación Asiática de Hispanistas. Pekín: Foreign Language Teaching and Research Press, pp. 3-21.

Briz, A. y M. Albelda (2013). "Una propuesta teórica y metodológica para el análisis de la atenuación lingüística en español y portugués. La base de un proyecto común (ES.POR.ATENUACIÓN)”, Onomazéin, 28, pp. 288-319: http://onomazein.letras.uc.cl/Articulos/N28/28-23_Briz_FINAL.pdf $(10 / 09 / 2016)$.

Briz, A. y M. Albelda (2013). "Una propuesta teórica y metodológica para el análisis de la atenuación lingüística en español y portugués. La base de un proyecto en común ES.POR.ATENUACIÓN”, Onomázein, 28, pp. 288-319.

Briz, A. y S. Pons (2010). "Unidades, marcadores discursivos y posición”. En Loureda, Ó. y E. Acín (eds.). Los estudios sobre marcadores del discurso en español, hoy. Madrid: Arco/Libros, pp. 327-358.

Caffi, C. (1999). “On mitigation”, Journal of Pragmatics, 31, pp. 881-909. http://www.sciencedirect. com/science/article/pii/S0378216698000988?via\%3Dihub (09/07/2016).

Caffi, C. (2000). La mitigazione. Un approccio pragmatico alla comunicazione nei contesti terapeutici. Pavia: C.L.U.

Calvet, L.J. (2004). Essais de linguistique. La langue est-elle une invention des linguistes? París: Plon.

Consejo de Europa (2002). Marco Común Europeo de Referencia para el aprendizaje, la enseñanza y la evaluación de lenguas. Instituto Cervantes-Ministerio de Educación, Cultura y Deporte. Madrid: Anaya.

Cresti, E. y M. Moneglia (2005). M. C-ORAL-ROM Integrated Reference Corpora for Spoken Romance Languages. Ámsterdam: John Benjamins.

Fraser, B. (1980). "Conversational mitigation", Journal of Pragmatics, 4, pp. 341-350. http://www. sciencedirect.com/science/article/pii/0378216680900296?via\%3Dihub (14/07/2016).

Fraser, B. (1990). "Perspectives on Politeness", Journal of Pragmatics, 14, pp. 219-236. http://www. sciencedirect.com/science/article/pii/037821669090081N?via\%3Dihub (07/07/2016).

Holmes, J. (1984). "Modifying illocutionary forcé”, Journal of Pragmatics, pp. 345-365.

Instituto Cervantes (2006), Plan curricular del Instituto Cervantes. Madrid: Instituto Cervantes: Biblioteca Nueva.

Lakoff, G. (1973). "Fuzzy Grammar and the Performance / Competence Game", Papers from the 9th Regional Meeting of the Chicago Linguistic Society. Chicago: Chicago University Press, pp. 271-291.

Meyer-Hermann, R. (1988). “Atenuación e intensificación (análisis pragmático de sus formas y funciones en el español hablado)”, Anuario de Estudios Filológicos, 11, pp. 275-290.

Sanchez Lobato, J. (1999). "Lengua y cultura. La tradición cultural hispánica”, Carabela, 45, pp. 5-26.

Sbisà, M. (2001). "Illocutionary force and degrees of strength in language use", Journal of Pragmatics, 33 (12), pp. 1791-1814.

Searle, J.R. (1980 [1969]). Actos de habla. Madrid: Cátedra.

Secchi, D. (2014). "De la comunicación oral dirigida a la autonomía comunicativa: la enseñanza de E/LE a través de corpus discursivos". Biblioteca Virtual RedELE (Red Electrónica de Didáctica del Español como Lengua Extranjera), Ministerio de Educación, Cultura y Deporte: http://www. mecd.gob.es/redele/Biblioteca-Virtual/2014/memorias-master/Daniel-Secchi.html (15/12/2016).

Villalba, C. (2015). "La ficha ES. VAR. ATENUACIÓN. ¿Un recurso adaptable a todos los géneros? El caso de los juicios orales", Textos en proceso, 1 (2), pp. 1-24. 